\title{
Emergence by design in self-assembling protein shells
}

Frank Sainsbury ${ }^{1,2, *}$

${ }^{1}$ Centre for Cell Factories and Biopolymers, Griffith Institute for Drug Discovery, Griffith University, Nathan, Queensland 4111, Australia.

${ }^{2}$ Synthetic Biology Future Science Platform, Commonwealth Scientific and Industrial Research Organisation (CSIRO), Brisbane, QLD 4001, Australia.

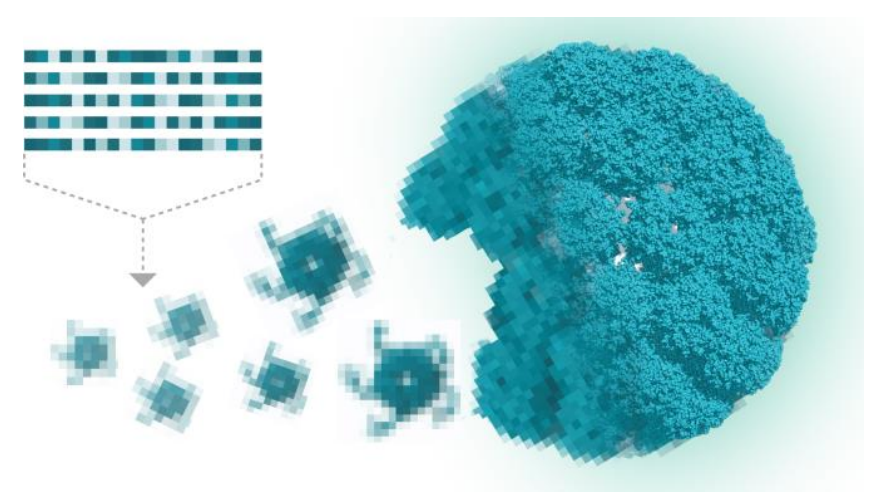

\section{Abstract}

The use of proteins and peptides as nanoscale components to generate new-to-nature physical entities holds great promise in biocatalysis, therapeutic or diagnostic delivery, and materials templating. The majority of functional particles have been based on existing structures found in nature. Developing biomimetic particles in this way takes advantage of highly evolved platforms for organisation or encapsulation of functional moieties, offering significant advantages in stoichiometry, multivalency and sequestration. However, novel assembly paradigms for the modular construction of macromolecular structures is now greatly expanding the functional diversity of protein-based nanoparticles in health and manufacturing. Kepiro et al., in this issue of ACS Nano, demonstrate the refinement of this concept, engineering the capacity for self-assembly such that it is integral to pore-forming peptide motifs, resulting in superior antibiotic activity of the selfassembled particle. Nature encodes multiple functions in proteins with exquisite efficiency and emulating this multiplicity may be the ultimate goal of biomimetic nanotechnologies. 
The majority of examples of neo-functional protein shells involve engineering existing structures found in nature like virus capsids or bacterial microcompartments. They give function to structure and there are numerous examples of how such bio-mimicking compartmentalisation can lead to remarkable properties. ${ }^{1-3}$ Less common outside of nature is taking a functional building block and using it to assemble a protein shell; to give structure to function (Figure 1). In both cases, emergent properties derived from the self-organisation of proteins or peptides into structures of different length scales set the assembly apart from the relatively limited function of individual building blocks. For example, the emergence of Förster resonance energy transfer between fluorescent proteins colocalized by their association with ${ }^{4}$ or encapsidation within virus capsids, ${ }^{5-6}$ or the de novo assembly of non-viral protein building blocks to encapsulate and protect nucleic acid. ${ }^{7}$ To the extent that bioengineering efforts are for the most part rational modifications to well characterised structures, such properties derive from a process that can be considered simple emergence; that which is without top-down feedback. ${ }^{8}$ In the case of giving function to structure, emergence is usually intentional. Unintentional emergence arises from the dependence of components on each other, reflecting the co-evolution of macromolecular assemblies in nature and the dependence of their self-assembly on feedback at the scale of the assembly units. ${ }^{9}$

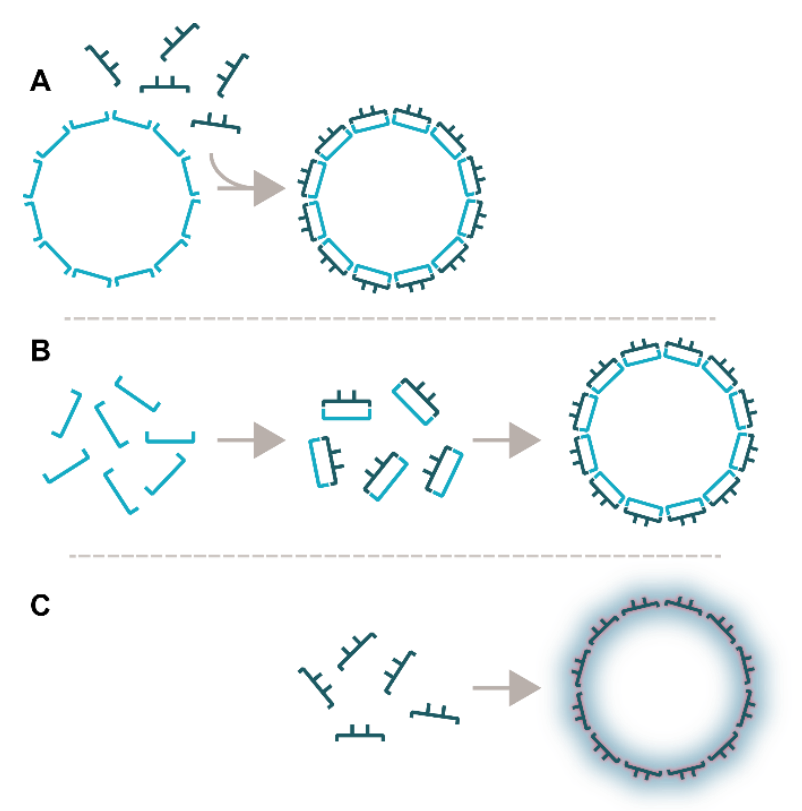

Figure 1. Generating protein shells with new and emergent functions. A) Adding function to an existing assembled structure; B) adding function to subunits of an existing structure; $\mathrm{C}$ ) integrating the capacity to assemble into self-assembling structures with functional building blocks. 


\section{A structure from function}

The paper by Kepiro et al. ${ }^{10}$ provides a striking example of unintentional emergence by design in the engineering of an innovative mode of antimicrobial delivery. Antimicrobial peptides have the potential to overcome resistance to existing antibiotics and represent a diverse class of molecules from which to draw on in the fight against bacterial pathogens. ${ }^{11}$ Despite strong antimicrobial activity, the necessity of reaching a local concentration threshold at the cell surface for bactericidal pore-formation is a drawback that limits the therapeutic window of antimicrobial peptides. The elegant approach described in this issue of ACS Nano addresses this problem by engineering the controlled aggregation of a pore-forming peptide into geometrically constrained nanoparticles, or pseudocapsids. The assembly of designer antimicrobial peptides provides the concentration of peptides required for pore formation in a single entity. In addition to this predicted function, the paper describes observations possibly related to the assembly of capsid-like architecture. An apparent preference of the pseudocapsids assembled from cationic peptides for the curved membrane at cell poles is likely related to the tendency of anionic lipids to accumulate in more highly curved membrane regions. ${ }^{12}$ Yet it is also reminiscent of virus-cell interactions where cell uptake of virions is dependent on permissive membrane domains with the necessary density of ligands. Importantly, in vivo assays in an insect model of methicillin-resistant Staphylococcus aureus (MRSA), demonstrates a persistent protective effect indicating considerably enhanced stability of the pseudocapsids relative to other bactericidal peptide monomers.

In Kepiro et al., ${ }^{10}$ a short aliphatic $\beta$-strand motif from lactoferrin, a host defence protein, is modified to facilitate inter-strand interactions, favouring the formation of anti-parallel $\beta$-sheets. By conjugation to a tri-functional adapter, the resulting triskelion peptide arms pair with the arms of another triskelion. Propagation of these $\beta$-sheet interactions generates a two-dimensional lattice that evidently closes into a sphere, minimising the free energy of 'sticky' $\beta$-strands stabilised by the interaction. The geometry of the resulting antimicrobial pseudocapsids is derived from the 3-fold rotational symmetry of the assembly units occupying the vertices of putative truncated icosahedra, with both pentagonal and hexagonal faces permitted. This accounts for the observed size polydispersity, reminiscent of icosahedral virus capsids composed of variations in the ratio of pentamers (always 12) and hexamers among the polyomaviruses. ${ }^{13-14}$ The regular organisation of subunits into lattices and multivalent display of functional moieties also leads to emergent properties of protein shells. There is a close relationship between the valency of virus attachment and cell uptake. ${ }^{15-16}$ For example, the multivalent presentation of receptor ligand mediates uptake, whereas a single subunit of the assembly does not. ${ }^{5}$ Similarly, aggregation of a short peptide with no 
measured activity as a monomer, into pseudocapsids composed of 100 s to 1000 s copies of the peptide, provides a valency of delivery that potentiate its antimicrobial effect. ${ }^{10}$

A characteristic of virus capsids and capsid-like protein shells is that relatively weak interactions between individual subunits combine, across the regular lattice of the entire shell to create a stable whole at thermodynamic equilibrium. The consequence is that such structures can persist under conditions that do not support assembly. Essential for many viruses that endure challenging environmental conditions to find the next host cell, it is also a considerable advantage of the antimicrobial pseudocapsids reported by Kepiro et al.. ${ }^{10}$ As the hydrophobic faces of the $\beta$-sheets would not be stable as a monolayer, the authors posit that a bilayer of the lattice is formed and molecular dynamics simulations of the relative stability of monolayer versus bilayer structures support this. Despite structural plasticity that accommodates size polymorphisms and enables rearrangements into pore-forming oligomers at lipid membranes, the distinctive bilayer structure results in pseudocapsids that are thermodynamically stable. ${ }^{10}$

\section{Best of both worlds}

Self-assembly of protein shells in nature provides protection, sequestration, and concentration for encapsulated cargos. Two well-documented examples are the protection and delivery of viral genomes to receptive subcellular locations by protein-based capsids, ${ }^{17}$ and the sequestration of essential biocatalysis by bacteria to the interior of protein-based compartments, so called bacterial micro compartments. ${ }^{18}$ Inspiration and genetic know-how have been borrowed from these systems to recreate self-assembling protein-based shells in the lab, permitting nanoscale control of heterologous biological, chemical and physical processes. The emergent properties in these systems are being developed to address significant challenges in health and manufacturing. Adenoassociated virus capsids are widely used for the delivery of heterologous genetic material. ${ }^{19}$ Encapsidation of multiple enzymes into recombinant capsids of P22 bacteriophage can enable enzymatic generation of molecular hydrogen. ${ }^{20}$ Densely arranged fluorophores, when geometrically organised by site-specific conjugation to Brome mosaic virus capsids, can be coaxed into quantum coherence manifest as cooperative radiative decay. ${ }^{21}$ However, and notwithstanding examples of polymorphism and tolerance to initial defects in natural protein shells, ${ }^{13,22}$ a considerable drawback of adapting existing structures for new functions is the susceptibility of the system to defects in single components. 
The molecular dynamics simulations performed by Kepiro et al., indicate that individual $\beta$-strand pairs can separate and re-form while the $\beta$-sheet network remains intact. This cooperative behaviour, where the interaction between assembly units is influenced by their place in the assemblage, is a hallmark of unintentional emergence ${ }^{8}$ and underpins the robustness of virus capsid assembly. ${ }^{9,22}$ The assembly of functional subunits into capsid-like structures is an encouraging step towards engineering synthetic protein shells with system-level redundancy. Further elucidating the molecular details of self-assembly pathways will allow for adaptation to different environments or applications for both natural and synthetic protein shells. Nanoscale control of reactions and interactions depends on near-atomic structural resolution of the system. While a range of oligomerizing triskelion conjugates of functional peptides have been designed to self-assemble, ${ }^{23}$ to date it has not been possible to empirically confirm their putative icosahedral geometry. On the other hand, the molecular precision with which natural protein shells assemble from much larger polypeptides is unmatched.

Several approaches are bridging the gap between the structural knowledge that supports the use of natural protein shells and the adaptability of newly designed subunits. For example, the combinatorial use of interacting domains ${ }^{24}$ and sophisticated computational design of structures from libraries of existing oligomeric proteins ${ }^{25}$ is leading to identification of novel self-assembling protein scaffolds. Protein engineering methods applied to shell-forming proteins, such as circular permutation, can expand the range of accessible polymorphic forms and control the position of addressable residues. ${ }^{26}$ And the directed evolution of natural and engineered protein shells is now sampling wider sequence space than was previously possible, generating novel forms ${ }^{27}$ and functions ${ }^{7,28-29}$. At the same time, the self-assembly of structured peptides including multimeric coiled-coil motifs ${ }^{30}$ and the $\beta$-sheet triskelions described by Kepiro et al., ${ }^{10}$ are leading to novel protein shells and questioning the requirement for regular subunit organisation for functional properties including stability. ${ }^{31}$ While the realm of naturally occurring protein shells would regard such irregular structures as kinetically trapped assembly defects or intermediates, the question is valid. The answer, of course, depends on the intended application.

In designing and using proteins shells, we hope to emulate nature. Among the myriad platforms developed from existing protein structure onto which new function is conferred, or the evolving design rules employed to assemble structures from functional peptides and motifs, deeper understanding of the emergent properties inherent to protein shell assemblies is developing. With this, so is our capacity to layer functionality within protein shells by genetic and chemical means. The work presented by Kepiro et al., highlights the potential of biomimicry in addressing some of the biggest challenges of our time, in this case, antibiotic resistance of bacterial pathogens. 


\section{References}

1. Aumiller, W. M.; Uchida, M.; Douglas, T., Protein cage assembly across multiple length scales. Chemical Society Reviews 2018, 47 (10), 3433-3469.

2. Edwardson, T. G. W.; Hilvert, D., Virus-Inspired Function in Engineered Protein Cages. Journal of the American Chemical Society 2019, 141 (24), 9432-9443.

3. Wen, A. M.; Steinmetz, N. F., Design of virus-based nanomaterials for medicine, biotechnology, and energy. Chemical Society Reviews 2016, 45 (15), 4074-4126.

4. Vogel, M.; Diez, M.; Eisfeld, J.; Nassal, M., In vitro assembly of mosaic hepatitis B virus capsid-like particles (CLPS): Rescue into CLPs of assembly-deficient core protein fusions and FRETsuited CLPs. FEBS Letters 2005, 579 (23), 5211-5216.

5. Dashti, N. H.; Abidin, R. S.; Sainsbury, F., Programmable In Vitro Coencapsidation of Guest Proteins for Intracellular Delivery by Virus-like Particles. ACS Nano 2018, 12 (5), 4615-4623.

6. O'Neil, A.; Prevelige, P. E.; Basu, G.; Douglas, T., Coconfinement of Fluorescent Proteins: Spatially Enforced Communication of GFP and mCherry Encapsulated within the P22 Capsid.

Biomacromolecules 2012, 13 (12), 3902-3907.

7. Butterfield, G. L.; Lajoie, M. J.; Gustafson, H. H.; Sellers, D. L.; Nattermann, U.; Ellis, D.; Bale, J. B.; Ke, S.; Lenz, G. H.; Yehdego, A.; Ravichandran, R.; Pun, S. H.; King, N. P.; Baker, D., Evolution of a designed protein assembly encapsulating its own RNA genome. Nature 2017, 552 (7685), 415-420.

8. Fromm, J. Types and Forms of Emergence arXiv e-prints [Online], 2005. https://ui.adsabs.harvard.edu/abs/2005nlin......6028F (accessed June 01, 2005).

9. Zandi, R.; Dragnea, B.; Travesset, A.; Podgornik, R., On virus growth and form. Physics Reports 2020.

10. Kepiro, I. E.; Marzuoli, I.; Hammond, K.; Ba, X.; Lewis, H.; Shaw, M.; Gunnoo, S. B.; De Santis, E.; Łapińska, U.; Pagliara, S.; Holmes, M. A.; Lorenz, C. D.; Hoogenboom, B. W.; Fraternali, F.; Ryadnov, M. G., Engineering Chirally Blind Protein Pseudocapsids into Antibacterial Persisters. ACS Nano 2019.

11. Wang, J.; Dou, X.; Song, J.; Lyu, Y.; Zhu, X.; Xu, L.; Li, W.; Shan, A., Antimicrobial peptides: Promising alternatives in the post feeding antibiotic era. Medicinal Research Reviews 2019, 39 (3), 831-859.

12. Epand, R. M.; Epand, R. F., Lipid domains in bacterial membranes and the action of antimicrobial agents. Biochimica et Biophysica Acta (BBA) - Biomembranes 2009, 1788 (1), 289-294.

13. Salunke, D. M.; Caspar, D. L.; Garcea, R. L., Polymorphism in the assembly of polyomavirus capsid protein VP1. Biophys J 1989, 56 (5), 887-900.

14. Nilsson, J.; Miyazaki, N.; Xing, L.; Wu, B.; Hammar, L.; Li, T. C.; Takeda, N.; Miyamura, T.; Cheng, R. H., Structure and assembly of a $T=1$ virus-like particle in BK polyomavirus. $J$ Virol 2005, 79 (9), 5337-5345.

15. Boulant, S.; Stanifer, M.; Lozach, P. Y., Dynamics of virus-receptor interactions in virus binding, signaling, and endocytosis. Viruses 2015, 7 (6), 2794-815.

16. Varga, C. M.; Wickham, T. J.; Lauffenburger, D. A., Receptor-mediated targeting of gene delivery vectors: Insights from molecular mechanisms for improved vehicle design. Biotechnology and Bioengineering 2000, 70 (6), 593-605.

17. Yamauchi, Y.; Helenius, A., Virus entry at a glance. Journal of Cell Science 2013, 126 (6), 1289-1295.

18. Kerfeld, C. A.; Aussignargues, C.; Zarzycki, J.; Cai, F.; Sutter, M., Bacterial microcompartments. Nature Reviews Microbiology 2018, 16 (5), 277-290.

19. Wang, D.; Tai, P. W. L.; Gao, G., Adeno-associated virus vector as a platform for gene therapy delivery. Nature Reviews Drug Discovery 2019, 18 (5), 358-378. 
20. Jordan, P. C.; Patterson, D. P.; Saboda, K. N.; Edwards, E. J.; Miettinen, H. M.; Basu, G.; Thielges, M. C.; Douglas, T., Self-assembling biomolecular catalysts for hydrogen production. Nature Chemistry 2016, 8 (2), 179-185.

21. Tsvetkova, I. B.; Anil Sushma, A.; Wang, J. C.-Y.; Schaich, W. L.; Dragnea, B., Radiation Brightening from Virus-like Particles. ACS Nano 2019, 13 (10), 11401-11408.

22. Lutomski, C. A.; Lyktey, N. A.; Zhao, Z.; Pierson, E. E.; Zlotnick, A.; Jarrold, M. F., Hepatitis B Virus Capsid Completion Occurs through Error Correction. Journal of the American Chemical Society 2017, 139 (46), 16932-16938.

23. Matsuura, K., Synthetic approaches to construct viral capsid-like spherical nanomaterials. Chemical Communications 2018, 54 (65), 8944-8959.

24. Wilson, C. J.; Bommarius, A. S.; Champion, J. A.; Chernoff, Y. O.; Lynn, D. G.; Paravastu, A. K.; Liang, C.; Hsieh, M.-C.; Heemstra, J. M., Biomolecular Assemblies: Moving from Observation to Predictive Design. Chemical Reviews 2018, 118 (24), 11519-11574.

25. Bale, J. B.; Gonen, S.; Liu, Y.; Sheffler, W.; Ellis, D.; Thomas, C.; Cascio, D.; Yeates, T. O.; Gonen, T.; King, N. P.; Baker, D., Accurate design of megadalton-scale two-component icosahedral protein complexes. Science 2016, 353 (6297), 389.

26. Azuma, Y.; Herger, M.; Hilvert, D., Diversification of Protein Cage Structure Using Circularly Permuted Subunits. Journal of the American Chemical Society 2018, 140 (2), 558-561.

27. Asensio, M. A.; Morella, N. M.; Jakobson, C. M.; Hartman, E. C.; Glasgow, J. E.; Sankaran, B.; Zwart, P. H.; Tullman-Ercek, D., A Selection for Assembly Reveals That a Single Amino Acid Mutant of the Bacteriophage MS2 Coat Protein Forms a Smaller Virus-like Particle. Nano Letters 2016, 16 (9), 5944-5950.

28. Brauer, D. D.; Hartman, E. C.; Bader, D. L. V.; Merz, Z. N.; Tullman-Ercek, D.; Francis, M. B., Systematic Engineering of a Protein Nanocage for High-Yield, Site-Specific Modification. Journal of the American Chemical Society 2019, 141 (9), 3875-3884.

29. Terasaka, N.; Azuma, Y.; Hilvert, D., Laboratory evolution of virus-like nucleocapsids from nonviral protein cages. Proceedings of the National Academy of Sciences 2018, 115 (21), 5432-5437. 30. Fletcher, J. M.; Harniman, R. L.; Barnes, F. R. H.; Boyle, A. L.; Collins, A.; Mantell, J.; Sharp, T. H.; Antognozzi, M.; Booth, P. J.; Linden, N.; Miles, M. J.; Sessions, R. B.; Verkade, P.; Woolfson, D. N., Self-Assembling Cages from Coiled-Coil Peptide Modules. Science 2013, 340 (6132), 595-599.

31. Mosayebi, M.; Shoemark, D. K.; Fletcher, J. M.; Sessions, R. B.; Linden, N.; Woolfson, D. N.; Liverpool, T. B., Beyond icosahedral symmetry in packings of proteins in spherical shells. Proceedings of the National Academy of Sciences 2017, 114 (34), 9014-9019. 